\title{
Application of He's Variational Iteration Method and Adomian Decomposition Method to Solution for the Fifth Order Caudrey-Dodd-Gibbon (CDG) Equation
}

\author{
Mehdi Safari \\ Department of Mechanical Engineering, Aligoodarz Branch, Islamic Azad University, Aligoodarz, Iran \\ E-mail:ms_safari2005@yahoo.com \\ Received June 14, 2010; revised June 26, 2011; accepted July 4, 2011
}

\begin{abstract}
In this work we use the He's variational iteration method and Adomian decomposition method to solution $\mathrm{N}$-soliton solutions for the fifth order Caudrey-Dodd-Gibbon (CDG) Equation.
\end{abstract}

Keywords: Variation Iteration Method, Adomian Decomposition Method, Caudrey-Dodd-Gibbon (CDG)

Equation

\section{Introduction}

The theory of solitary waves has attracted much interest in recent years for treatment of PDEs describing nonlinear and evolution concepts. Nonlinear phenomena appear in many areas of scientific fields such as solid state physics, plasma physics, fluid dynamics, mathematical biology and chemical kinetics. The nonlinear problems are characterized by dispersive effects, dissipative effects, convection-advection, and diffusion process. A broad class of analytical solutions methods, such as inverse scattering method, Ba"cklund transformation method, Hirota's bilinear scheme [1-6], Hereman's method [7,8], pseudo spectral method, Jacobi elliptic method, Painleve' analysis [9], and other methods, were used to handle these problems. However, some of these analytical solutions methods are not easy to use because of the tedious work that it requires. This paper is concerned with the multiple-soliton solutions of the fifth order nonlinear Caudrey-Dodd-Gibbon (CDG) equation [10,11]

$$
u_{t}+u_{x x x x}+30 u u_{x x x}+30 u_{x} u_{x x}+180 u^{2} u_{x}=0
$$

with $u(x, t)$ is a sufficiently often differentiable function. It is well-known that this equation is completely integrable. This means that it has multiple-soliton solutions. The CDG equation possesses the Painleve' property as proved by Weiss in [9]. A useful study is introduced in [9] using the Painleve' property and the Ba"cklund transformation in handling the CDG equation and other equations as well. It was found in [9] that the
CDG Equation (1) has the Backlund transformation

$$
u=\frac{\partial^{2}}{\partial x^{2}} \ln \Phi+u_{2}
$$

where $u_{2}$ satisfies the CDG equation, and

$$
u 2=-\frac{1}{6} \frac{\Phi_{x x x}}{\Phi_{x}}
$$

and

$$
\frac{\Phi_{t}}{\Phi_{x}}+\frac{\partial^{2}}{\partial x^{2}}\{\Phi ; x\}+4\{\Phi ; x\}^{2}=0
$$

The last two equations can be expressed as the Lax pair

$$
\begin{gathered}
\Phi_{x x x}+6 u 2 \Phi_{x x x}=0 \\
\Phi_{t}+18 u_{2 x} \Phi_{x x}+6\left(6 u_{2}^{2}-u 2 x x\right) \Phi_{x}=0
\end{gathered}
$$

The objective of this work is to further complement other studies related to the CDG equation. The tanh method [12-17], and the tanh-coth method [18,19] will be used to stress its power in the determination of singlesoliton solution and other travelling wave solutions. We aim to use Adomian decomposition method and variation iteratin method to solve this equation.

\section{Basic Idea of He's Variational Iteration Method}

To clarify the basic ideas of VIM, we consider the fol- 
lowing differential equation:

$$
L u+N u=g(t)
$$

where $L$ is a linear operator, $N$ a nonlinear operator and $g(t)$ an inhomogeneous term.

According to VIM, we can write down a correction functional as follows:

$$
u_{n+1}(t)=u_{n}(t)+\int_{0}^{t} \lambda\left(L u_{n}(\tau)+N \tilde{u}_{n}(\tau)-g(\tau)\right) \mathrm{d} \tau
$$

where $\lambda$ is a general Lagrangian multiplier which can be identified optimally via the variational theory. The subscript $n$ indicates the $n$th approximation and $\tilde{u}_{n}$ is considered as a restricted variation $\delta \tilde{u}_{n}=0$.

\section{a. VIM Implement for this Equation:}

We first consider the application of VIM CaudreyDodd-Gibbon(CDG) equation:

$$
u_{t}+u_{x x x x}+30 u u_{x x x}+30 u_{x} u_{x x}+180 u^{2} u_{x}=0
$$

with the initial conditions of:

$$
u_{0}(x, t)=\mu^{2} \sec h^{2}(\mu x)
$$

To earn general Lagrangian multiplier $(\lambda)$ we put coeficient of $u_{t}$ or $u_{x}$ equal zero.where prime indicates a differential with respect to $x$ and dot denotes a differential with respect to $t$. We earn $\lambda$ respect to $t$. After some calculations, we obtain the following stationary conditions in Equations(10a) and (10b):

$$
\begin{gathered}
1+\left.\lambda(\tau)\right|_{\tau=t}=0 \\
\lambda(\tau)=-1
\end{gathered}
$$

Its correction variational functional in $x$ and $t$ can be expressed, respectively, as follows:

$$
\begin{aligned}
u_{n+1}(x, t) & =u_{n}(x, t)+\int_{0}^{t} \lambda\left(\frac{\partial}{\partial \tau} u_{n}(x, t)+\frac{\partial^{5}}{\partial x^{5}} u_{n}(x, t)\right. \\
& +30 \frac{\partial}{\partial x} u_{n}(x, t) \frac{\partial^{2}}{\partial x^{2}} u_{n}(x, t)+30 u_{n}(x, t) \\
\cdot & \left.\frac{\partial^{3}}{\partial x^{3}} u_{n}(x, t)+180 u_{n}(x, t)^{2} \frac{\partial}{\partial x} u_{n}(x, t)\right) \mathrm{d} \tau
\end{aligned}
$$

We start with the initial approximation of $u(x, 0)$ given by Equation (4). Using the above iteration formulas (9), we can directly obtain the other components as follows:

$$
\begin{aligned}
u_{1}(x, t) & =\frac{\mu^{2}\left(\cosh (\mu x)+32 \mathrm{t} \mu^{5} \sinh (\mu x)\right)}{\cosh ^{3}(\mu x)} \\
u_{2}(x, t) & =\frac{1}{\cosh (\mu \mathrm{x})^{10}} \mu^{2}\left(\cosh (\mu \mathrm{x})^{8}\right. \\
& +32 \mu^{5} \sinh (\mu \mathrm{x}) \operatorname{tcosh}(\mu \mathrm{x})^{7} \\
& +163840 \mu^{15} \sinh (\mu \mathrm{x}) \mathrm{t}^{3} \cosh (\mu \mathrm{x})^{5} \\
& -614400 \mu^{15} \sinh (\mu \mathrm{x}) \mathrm{t}^{3} \cosh (\mu \mathrm{x})^{3} \\
& -7372800 \mu^{20} \mathrm{t}^{4} \cosh (\mu \mathrm{x})^{2} \\
& +2949120 \mu^{20} \mathrm{t}^{4} \cosh (\mu \mathrm{x})^{4}+4423680 \mu^{20} \mathrm{t}^{4} \\
& +491520 \mu^{15} \sinh (\mu \mathrm{x}) \mathrm{t}^{3} \cosh (\mu \mathrm{x}) \\
& +512 \mu^{10} \mathrm{t}^{2} \cosh (\mu \mathrm{x})^{8}-768 \mu^{10} \mathrm{t}^{2} \cosh (\mu \mathrm{x})^{6}
\end{aligned}
$$

and continue then we show the last result in Figure 1(a).

\section{Basic Idea of Adomian Decomposition Method}

We begin with the equation

$$
L u+R(u)+F(u)=g(t)
$$

where $L$ is the operator of the highest-ordered derivatives with respect to $\mathrm{t}$ and $R$ is the remainder of the linear operator. The nonlinear term is represented by $F(u)$. Thus we get

$$
L u=g(t)-R(u)-F(u)
$$

The inverse $L^{-1}$ is assumed an integral operator given by

$$
L^{-1}=\int_{0}^{t}(\cdot) \mathrm{d} t
$$

The operating with the operator $L^{-1}$ on both sides of Equation (15) we have

$$
u=f_{0}+L^{-1}(g(t)-R(u)-F(u))
$$

where $f_{0}$ is the solution of homogeneous equation

$$
L u=0
$$

involving the constants of integration. The integration constants involved in the solution of homogeneous Equation (18) are to be determined by the initial or boundary condition according as the problem is initial-value problem or boundary-value problem.

The ADM assumes that the unknown function $u(x, t)$ 
can be expressed by an infinite series of the form

$$
u(x, t)=\sum_{n=0}^{\infty} u_{n}(x, t)
$$

and the nonlinear operator $F(u)$ can be decomposed by an infinite series of polynomials given by

$$
F(u)=\sum_{n=0}^{\infty} A_{n}
$$

where $u_{n}(x, t)$ will be determined recurrently, and $A_{n}$ are the so-called polynomials of $u_{0}, u_{1}, \cdots, u_{n}$ defined by

$$
A_{n}=\frac{1}{n !} \frac{\mathrm{d}^{n}}{\mathrm{~d} \lambda^{n}}\left[F\left(\sum_{n=0}^{\infty} \lambda^{i} u_{i}\right)\right]_{\lambda=0}, \quad n=0,1,2, \cdots
$$

It is now well known in the literature that these polynomials can be constructed for all classes of nonlinearity according to algorithms set by Adomian $[10,15]$ and recently developed by an alternative approach in $[8,9$, 16,17].

\section{b. ADM Implement for This Equation}

We solve this equation

$$
\begin{aligned}
u_{n+1}(x, t) & =\int_{0}^{t}\left(-\frac{\partial^{5}}{\partial x^{5}} u_{n}(x, \tau)-30 \frac{\partial}{\partial x} u_{n}(x, \tau)\right. \\
& \cdot \frac{\partial^{2}}{\partial x^{2}} u_{n}(x, \tau) 30 u_{n}(x, \tau) \frac{\partial^{3}}{\partial x^{3}} u_{n}(x, \tau) \\
& \left.-180 u_{n}(x, \tau)^{2} \frac{\partial}{\partial x} u_{n}(x, \tau)\right) \mathrm{d} \tau
\end{aligned}
$$

We solve with this way CDG equation and continue

$$
\begin{aligned}
u_{1}(x, t)=\frac{32 \mu^{7} t \sinh (\mu x)}{\cosh ^{3}(\mu x)} \\
u_{2}(x, t)=\frac{1}{\cosh (\mu x)^{10}} \cdot\left(1 2 8 \mu ^ { 1 2 } \mathrm { t } ^ { 2 } \left(23040 \mu^{10} \mathrm{t}^{2} \cosh (\mu x)^{4}\right.\right. \\
-57600 \mu^{10} \mathrm{t}^{2} \cosh (\mu x)^{2}+34560 \mu^{10} \mathrm{t}^{2} \\
+1280 \mu^{5} \sinh (\mu x) \operatorname{tcosh}(\mu x)^{5} \\
-7680 \mu^{5} \sinh (\mu x) \operatorname{tcosh}(\mu x)^{3} \\
+7680 \mu^{5} \sinh (\mu x) \operatorname{tcosh}(\mu x) \\
+4 \cosh (\mu x)^{8}-126 \cosh (\mu x)^{6} \\
\left.+420 \cosh (\mu x)^{4}-315 \cosh (\mu x)^{2}\right)
\end{aligned}
$$

$$
\begin{aligned}
u(x, t) & =\mu^{2} \operatorname{sech}^{2}(\mu x)+\frac{32 \mu^{7} \mathrm{t} \sinh (\mu x)}{\cosh ^{3}(\mu x)} \\
& +\frac{1}{\cosh (\mu x)^{10}}\left(1 2 8 \mu ^ { 1 2 } \mathrm { t } ^ { 2 } \left(23040 \mu^{10} \mathrm{t}^{2} \cosh (\mu x)^{4}\right.\right. \\
& -57600 \mu^{10} \mathrm{t}^{2} \cosh (\mu x)^{2}+34560 \mu^{10} \mathrm{t}^{2} \\
& +1280 \mu^{5} \sinh (\mu x) \operatorname{tcosh}(\mu x)^{5} \\
& -7680 \mu^{5} \sinh (\mu x) \operatorname{tcosh}(\mu x)^{3} \\
& +7680 \mu^{5} \sinh (\mu x) \operatorname{tcosh}(\mu x) \\
& +4 \cosh (\mu x)^{8}-126 \cosh (\mu x)^{6} \\
& \left.+420 \cosh (\mu x)^{4}-315 \cosh (\mu x)^{2}\right)
\end{aligned}
$$

We sum $u_{i}$ in Equation (25) and earn the results according to $\mu_{i}=1,1.5,2,3$ in Figures 1-4.

We compared 2-D figures of VIM and ADM for different values of $\mu$ in Figure 5 .

\section{Conclusions}

In this paper, He's variational iteration method has been successfully applied to find the solution CDG equations.

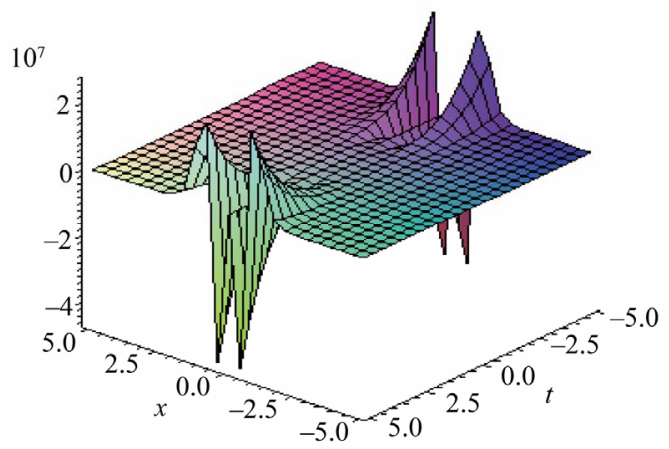

(a)

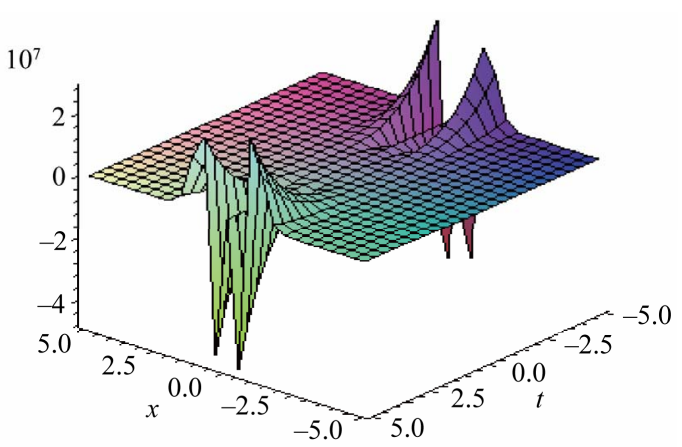

(b)

Figure 1. For the solitary wave solution with the first initial conditions (4) of Equation (1), VIM result for $u(x, t)$ is, respectively (b) and $\mathrm{ADM}(\mathrm{b})$, with $\mu=1$. 


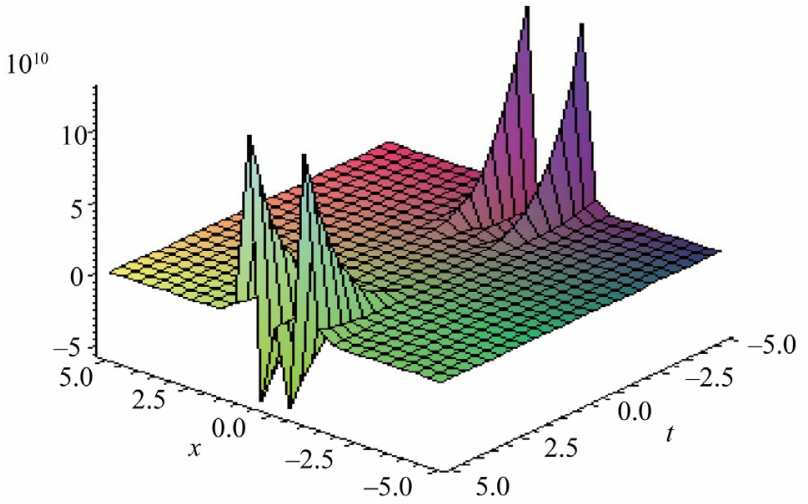

(a)

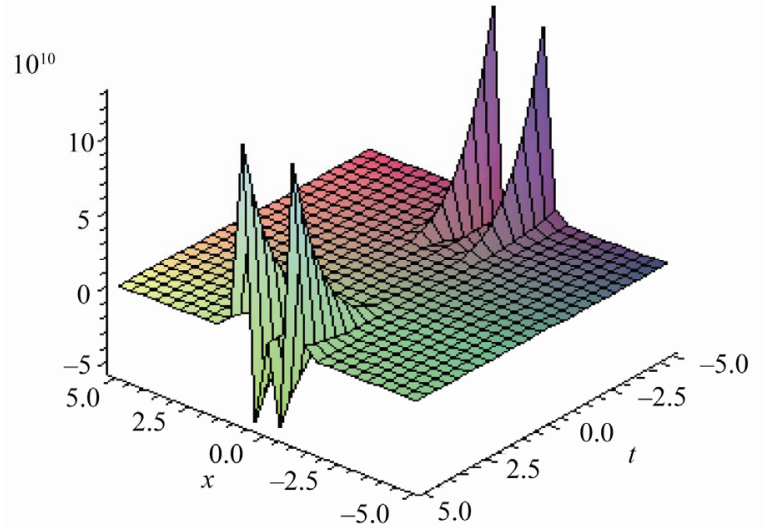

(b)

Figure 2. For VIM result for $u(x, t)$ is, respectively (1b) and $\operatorname{ADM}(1 b)$, with $\mu=1.5$.

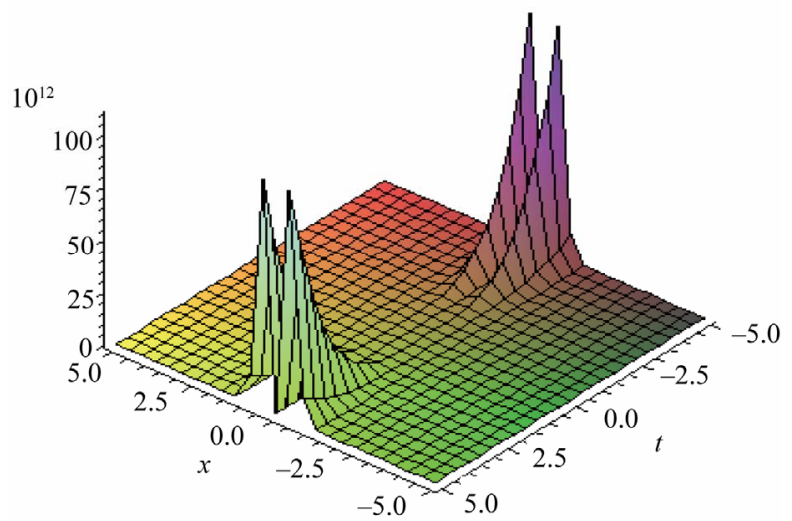

(a)

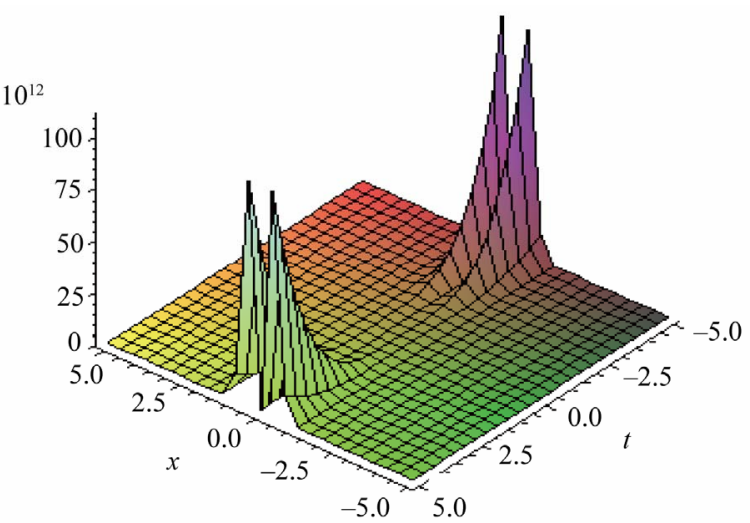

(b)

Figure 3. VIM result for $u(x, t)$ is, respectively (1b) and ADM(1b), with $\mu=2$.

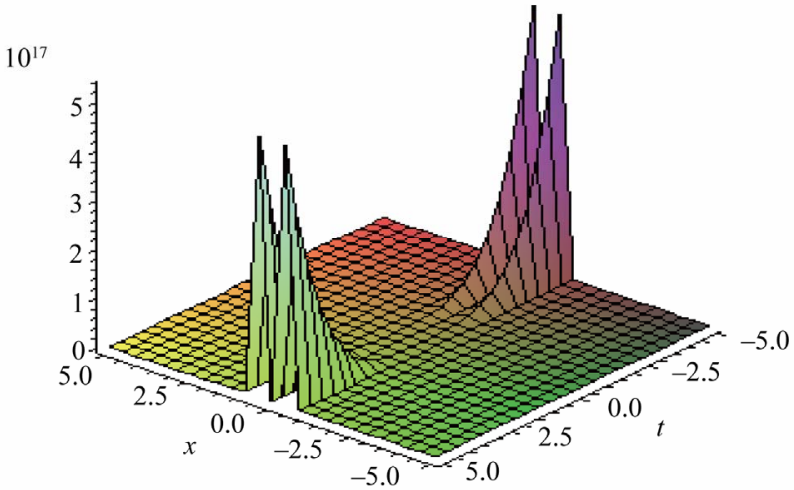

(a)

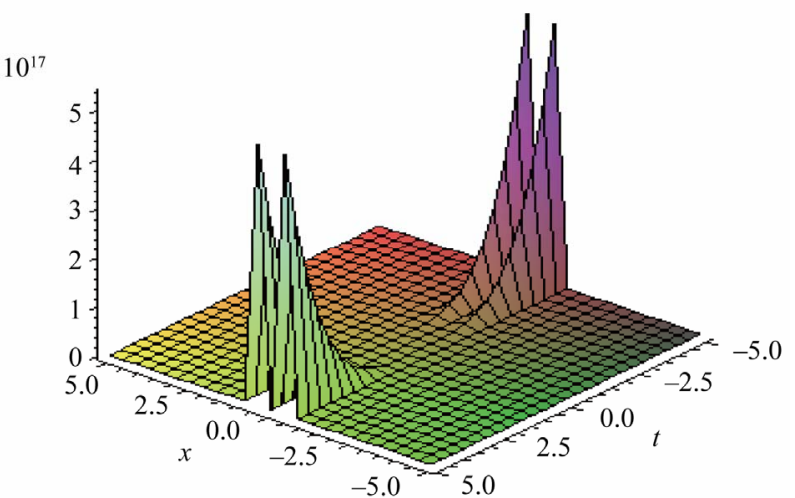

(b)

Figrue 4. VIM result for $u(x, t)$ is, respectively (1) and $\operatorname{ADM}(1)$, with $\mu=3$.

Both of methods show that the results are in excellent agreement with toghether and the obtained solutions are shown graphically. In our work, we use the Maple Package to calculate the functions obtained from the varia- tional iteration method and adomian decomposition method. An interesting point about ADM is that with the fewest number of iterations or even in some cases, once, it can converge to correct results. 


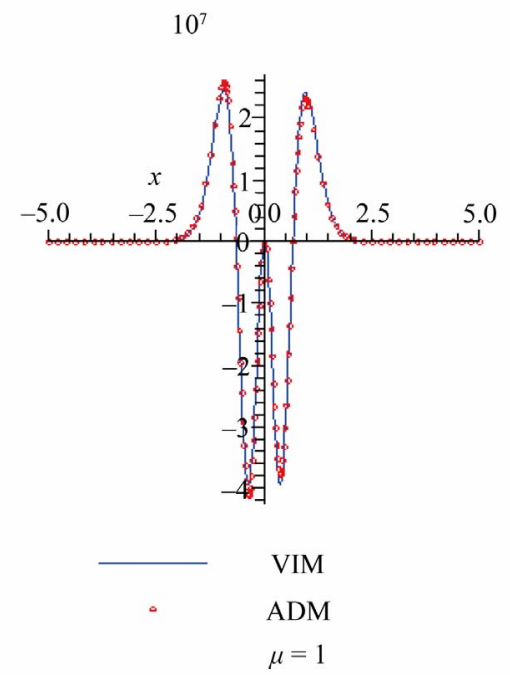

$10^{13}$

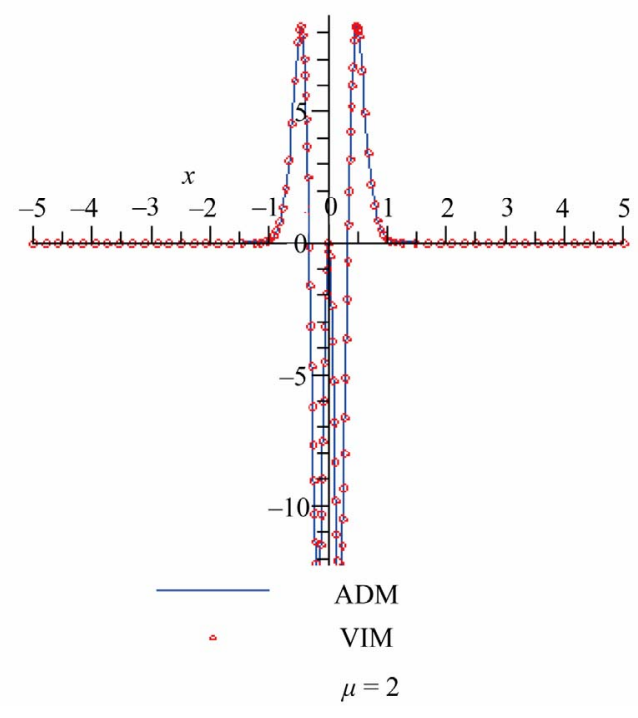

$10^{11}$

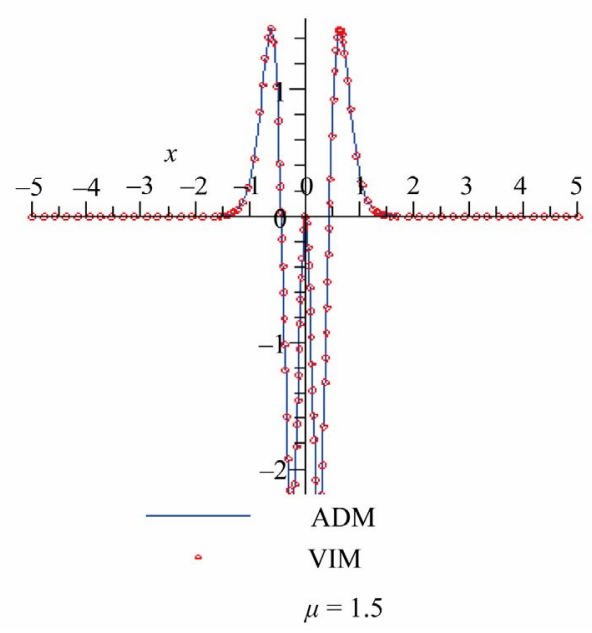

$10^{16}$

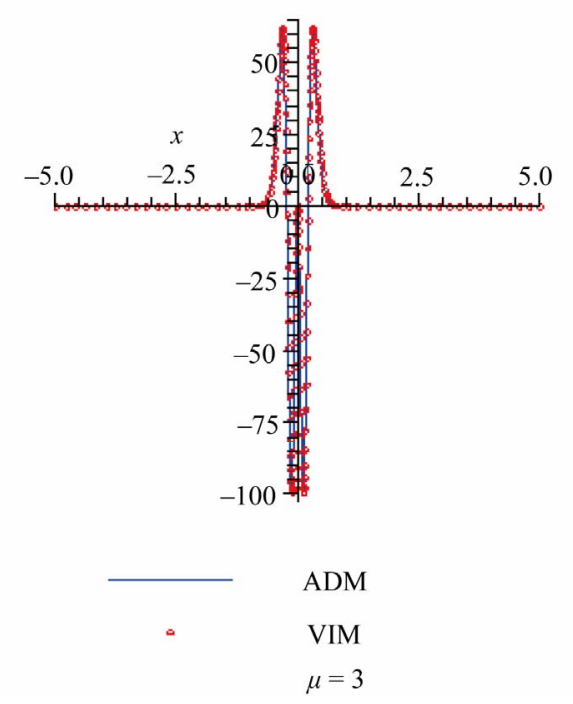

Figure 5. Comparison 2-D figures of VIM and ADM for different values of $\mu$.

\section{References}

[1] R. Hirota, “The Direct Method in Soliton Theory,” Cambridge University Press, Cambridge, 2004.

[2] R. Hirota, "Exact Solutions of the Korteweg-de Vries Equation for Multiple Collisions of Solitons,” Physical Review Letters, Vol. 27, No. 18, 1971, pp. 1192-1194. doi:10.1103/PhysRevLett.27.1192

[3] R. Hirota, "Exact Solutions of the Modified Korteweg-de Vries Equation for Multiple Collisions of Solitons," Journal of the Physical Society of Japan, Vol. 33, No. 5, 1972, pp. 1456-1458. doi:10.1143/JPSJ.33.1456

[4] R. Hirota, "Exact Solutions of the Sine-Gordon Equation for Multiple Collisions of Solitons," Journal of the Physical Society of Japan, Vol. 33, No. 5, 1972, pp. 14591463. doi:10.1143/JPSJ.33.1459
[5] J. Hietarinta, “A Search for Bilinear Equations Passing Hirota's Three-Soliton Condition. I. KdV-Type Bilinear Equations," Journal of Mathematical Physics, Vol. 28, No. 8, 1987, pp. 1732-1742. doi:10.1063/1.527815

[6] J. Hietarinta, "A Search for Bilinear Equations Passing Hirota's Three-Soliton Condition. II. mKdV-Type Bilinear Equations," Journal of Mathematical Physics, Vol. 28, No. 9, 1987, pp. 2094-2101. doi:10.1063/1.527421

[7] W. Hereman and W. Zhaung, "Symbolic Software for Soliton Theory," Acta Applicandae Mathematicae, Physics Letters A, Vol. 76, 1980, pp. 95-96.

[8] W. Hereman and A. Nuseir, "Symbolic Methods to Construct Exact Solutions of Nonlinear Partial Differential Equations," Mathematics and Computers in Simulation, Vol. 43, 1997, pp. 13-27. doi:10.1016/S0378-4754(96)00053-5 
[9] J. Weiss, "On Classes of Integrable Systems and the Painleve' Property,” Journal of Mathematical Physics, Vol. 25, No. 1, 1984, pp. 13-24. doi:10.1063/1.526009

[10] P. J. Caudrey, R. K. Dodd and J. D. Gibbon, “A New Heirarchy of Korteweg-de Vries Equations,” Proceedings of the Royal Society A, Vol. 351, 1976, pp. 407-422. doi:10.1098/rspa.1976.0149

[11] R. K. Dodd and J. D. Gibbon, "The Prolongation Structure of a Higher Order Korteweg-de Vries Equations,” Proceedings of the Royal Society A, Vol. 358, 1977, pp. 287-300.

[12] W. Malfliet, “The Tanh Method: A Tool for Solving Certain Classes of Nonlinear Evolution and Wave Equations," Journal of Computational and Applied Mathematics, Vol. 164-165, 2004, pp. 529-541. doi:10.1016/S0377-0427(03)00645-9

[13] W. Malfliet, "Solitary Wave Solutions of Nonlinear Wave Equations," American Journal of Physics, Vol. 60, No. 7, 1992, pp. 650-654. doi:10.1119/1.17120

[14] W. Malfliet and W. Hereman, "The Tanh Method: I. Exact Solutions of Nonlinear Evolution and Wave Equations,” Physica Scripta, Vol. 54, 1996, pp. 563-568. doi:10.1088/0031-8949/54/6/003

[15] W. Malfliet and W. Hereman, "The Tanh Method: II. Perturbation Technique for Conservative Systems,” Physica Scripta, Vol. 54, 1996, pp. 569-575. doi:10.1088/0031-8949/54/6/004

[16] A. M. Wazwaz, "The Tanh Method for Travelling Wave Solutions of Nonlinear Equations," Applied Mathematics and Computation, Vol. 154, No. 3, 2004, pp. 713-723. doi:10.1016/S0096-3003(03)00745-8

[17] A. M. Wazwaz, "Partial Differential Equations: Methods and Applications,” Balkema Publishers, The Netherlands, 2002.

[18] A. M. Wazwaz, "The Extended Tanh Method for New Solitons Solutions for Many Forms of the Fifth-Order KdV Equations," Applied Mathematics and Computation, Vol. 184, No. 2, 2007, pp. 1002-1014. doi:10.1016/j.amc.2006.07.002

[19] A. M. Wazwaz, "The Tanh-Coth Method for Solitons and Kink Solutions for Nonlinear Parabolic Equations,” $A p$ plied Mathematics and Computation, Vol. 188, 2007, pp. 1467-1475. doi:10.1016/j.amc.2006.11.013 